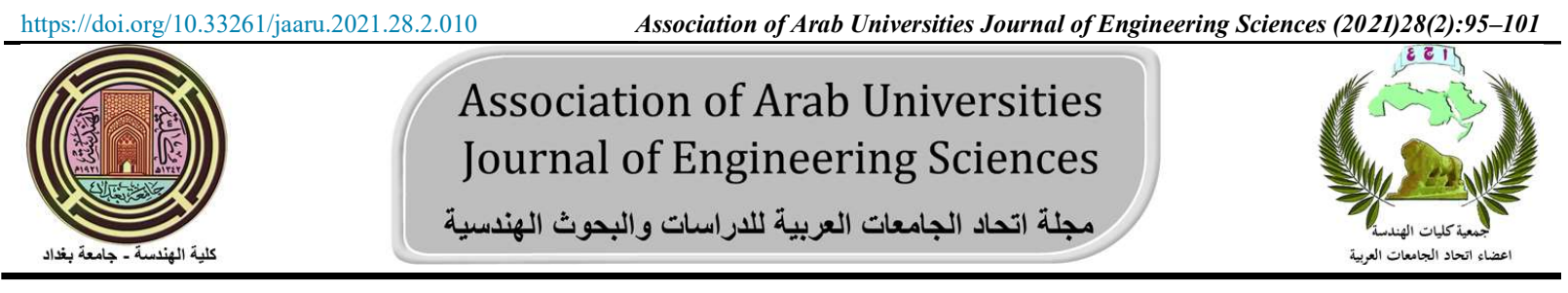

دراسة تأثير مادة كبريتات الباريوم كمادة مالئة على الخواص الفيزيائية والميكانيكية لمركبات المطاط النتريلي

ali.alhaidar315@gmail.com هندسة المواد التطبيقية ، قسم هنسة المعدات والآليات ،كلبة المهنسة الثقنية ،جامعة طرطوس ، سوريا، alimali1968@yahoo.com، قسم تقانة الأغنية ، كلية العندسة الثقنية ، جامعة طرطوس ، سورية mayssaali@yahoo.fr قسم العدات والآليات ،كلية المندسة التقنية، جامعة طرطوس ، سوريا، alialhaidar315@gmail.com الباحث المثل : علي غسان الحيبر، الايبيل: شر في:30 حزيران 2021

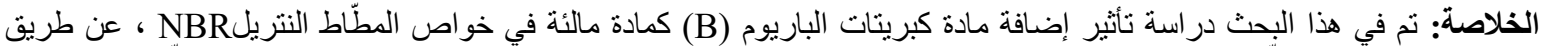

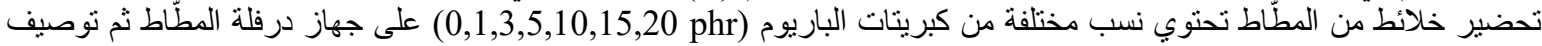

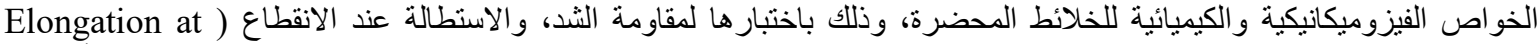

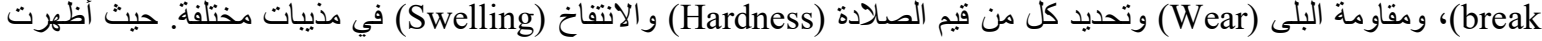

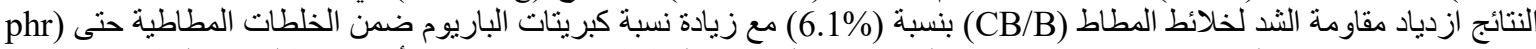

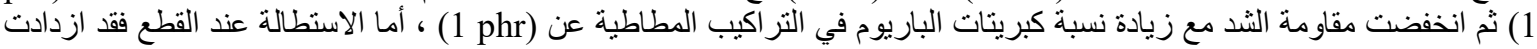

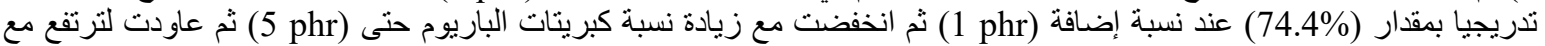

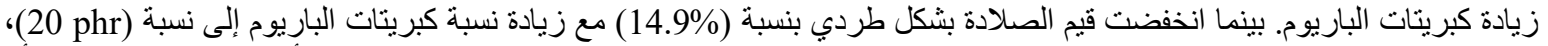

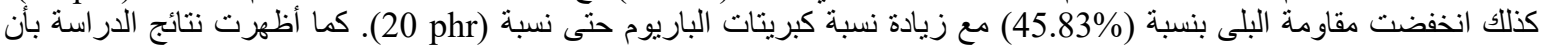

انتفاخ العينات التي تحتوي على الكربون الأسود فقط (C45B0) كانت منخفضة للغاية مقارنة بالعينات تحنوي على كبريتات الباريوم.

A - مو ادّ مالئة تضاف لتحسين الخو اصنّ الميكانيكيّة للمطَّاط وتقسم إلى:

$$
\text { الكلمات الرئيسية - الفلكنة، كبرينات الباريوم، المطاط، الكربون الأسود، الدرفلة. }
$$

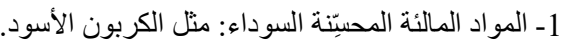

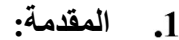

2 ـ الموادّ المالئة المحسيّنة البيضساء: مثل مركَبات السيليس (Sio

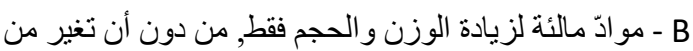

خو اصنّ المطّاط الميكانيكيّة.

المطاط النتريلي (NBR) ويعتبر من أنواع المطاط الصناعي ويحصل عليه

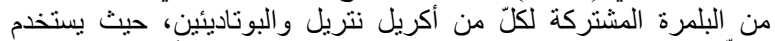

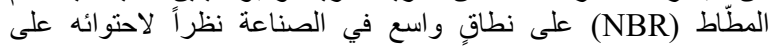
مونومير

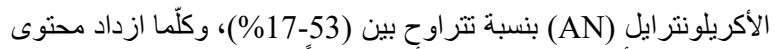

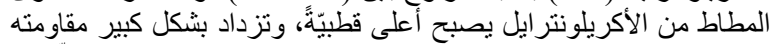

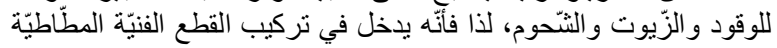

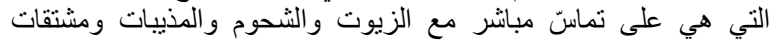

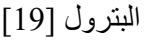

كنلك قام الباحث (Alneamah) [5] عام (2015) بذر اسة وتحسين الثباتية

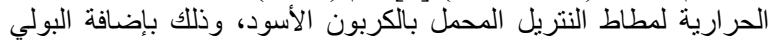

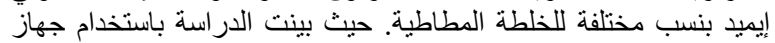
التحليل الوزني الحراري (TGA) والتحليل الديكانيكي الديناميكي

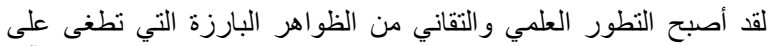

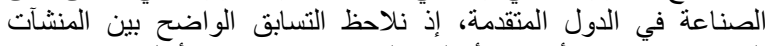

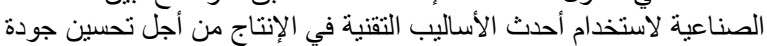

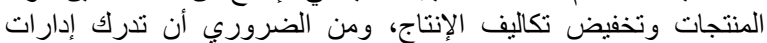

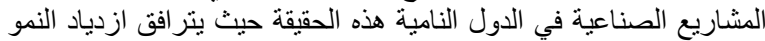

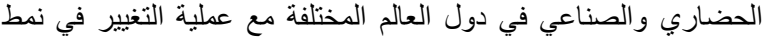

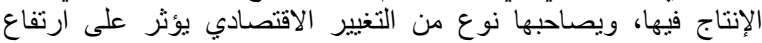

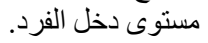

حيث ينم استخدام أنواع مختلفة من المواد المالئة والهجينة مع الكربون

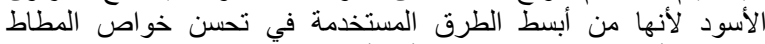
النتريلي و أقل الطر ائق كلفة، حيث أنها أسهمت في إنتاج عدد من السلع ذات التهات

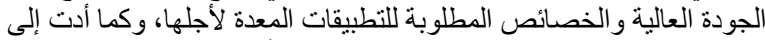

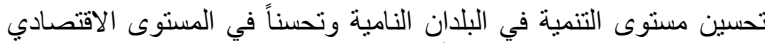

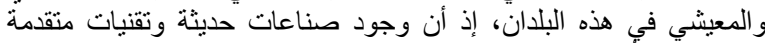

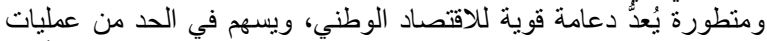
الاستير اد من الخارج وتوفير في القطع الاجنبي و الاكتفاء الذاتي صناعياً.

كما تعدّ المو اد المالئة من أهم المو اد التي تضاف للمطاط لتحسين خو اصده

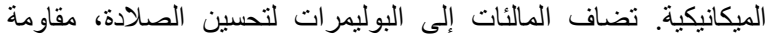

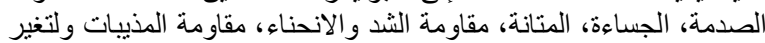
الخواصنّ الكهربائية للبوليمر ات [1] ـ وتقسم المالئات قسمين]ة]

1726-4081@ 2021The author(s). Published by Association of Arab Universities Journal of Engineering Sciences. This is an open access article under the CC BY-NC license (https://creativecommons.org/licenses/by-nc/4.0/). 
$.(375+20) \mathrm{kg} / \mathrm{m} 3$

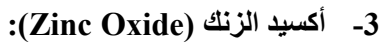

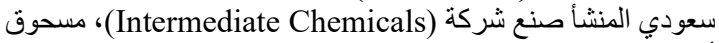
أبيض اللون، درجة النقاء (\% (99.9)، الكثافة الظاهرية 1.8 - 1.5 (1.5)

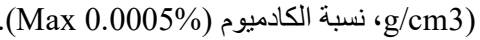

4- حمض الثمع (Stearic Acid):

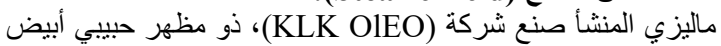

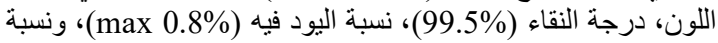

الحمض (195 min (195، (ل).

5- - مضاد الأكسدة (Antioxidants): استخدم في البحث مضاد الاكدة الأكسدة (I010NA (IPPD)، بريطاني

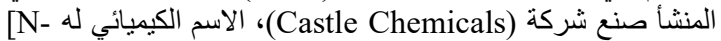
isopropyl-N'-phenyl-p-phenylenediamine] حبيبي بني داكن، نسبة الرماد Max (G) M \% 0.2)، درجة النقاء . [0.97 (GC) $\geq \%$ ]

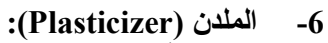
زيت ثنائي أوكتيل فتالات (Dioctyl Phthalate)

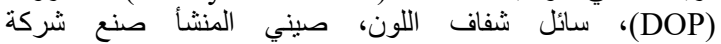
(Zhengzhou San Techchem)

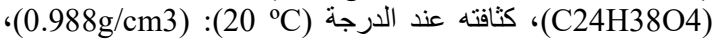
النقاوة (Min 99.5\%)، نقطة الوميض (Min $196{ }^{\circ} \mathrm{C}$ (M) : (24).

7- المواد المسرعة لعملية القلكنة (Accelerators):

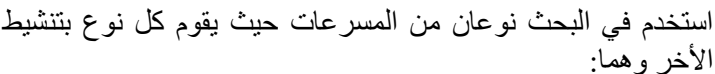

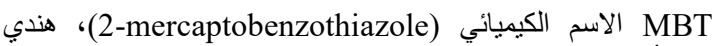

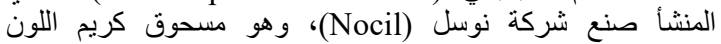

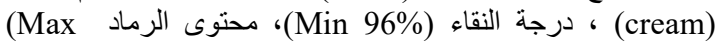

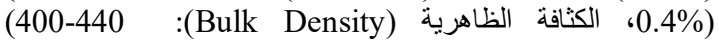

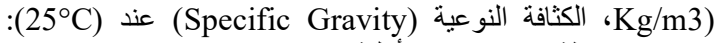

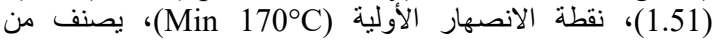

المسر عات شبه فائق السر عة (Semi ultra-fast accelerator)،

، (Tetramethyl thiuram disulfide) الاسم الكيميائي TMTD

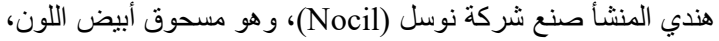

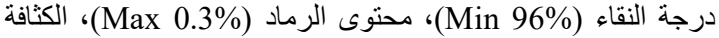

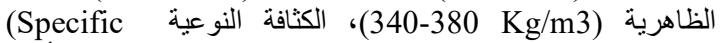
Gravity)

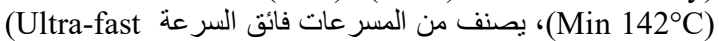
accelerator)

8 - كبريتات الباريوم (Barium sulfate):

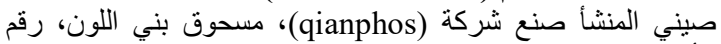

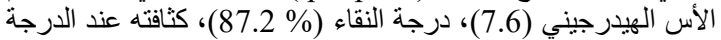

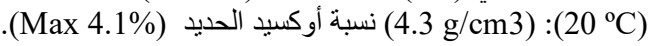

9- مواصفات الديزل (Diesel)

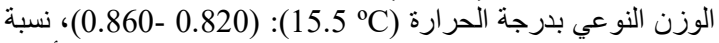

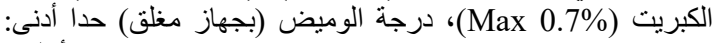
(Winter: $55{ }^{\circ} \mathrm{C}$ - Summer $\left.60{ }^{\circ} \mathrm{C}\right)$

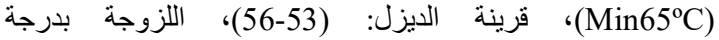

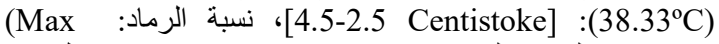

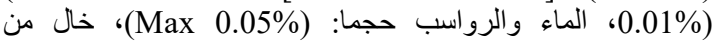
(الحموض و القلويات، وزن راسب الكربون (كونر ادسون): Max 0.1)
للمركبات المحضرة استقرارًا حراريًا أفضل بكثير. ارتفعت (DMTA)

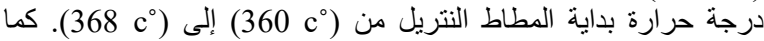

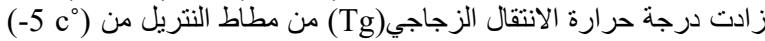

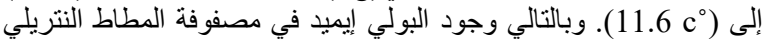
يزيد من الاستقرار الحراري.

كما قام الباحث (Senthilvel) [17] عام (2016) بدراسة نأثثير مواد

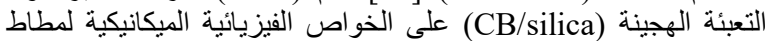

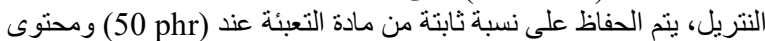

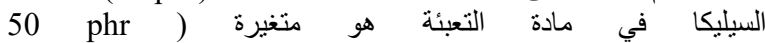

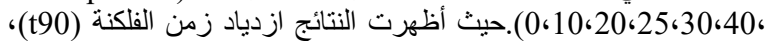

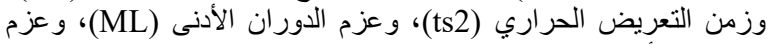

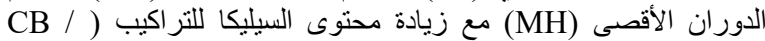

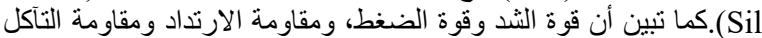

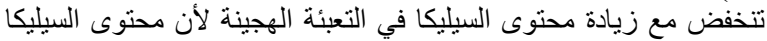
يجعل المركبات أقل صلابة.

وقد درس عدد كبير من الباحثين تأثير تعديل المطاط النتريلي, حيث قام النقام

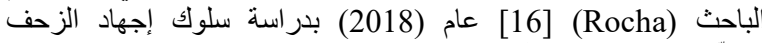

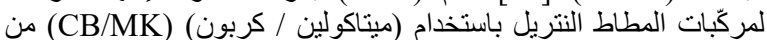

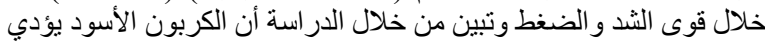

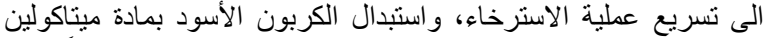

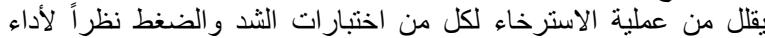

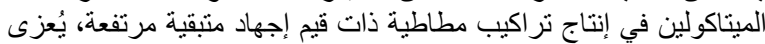

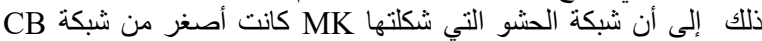

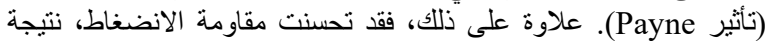

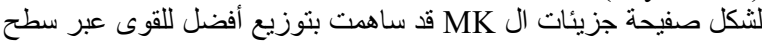

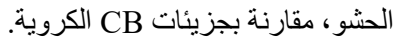

تكمن أهمية البحث الحصول على منتجات من المطاط النتريلي NBR ذات

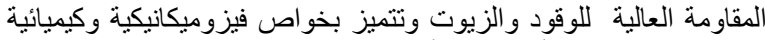

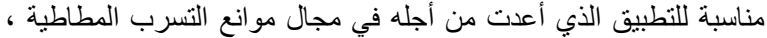

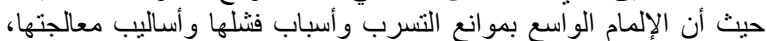

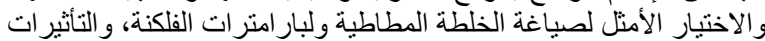

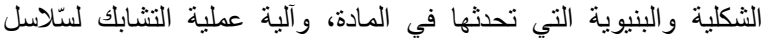

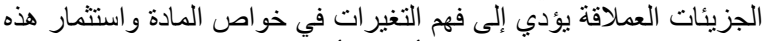
الخو اص بما يناسب التطبيق الذبي أعدّت لأجله.

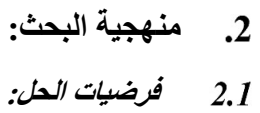

أن المواد المالئة لها دوراً كبير ا في تحسن الخصائص الميكانيكية الفيزيائية

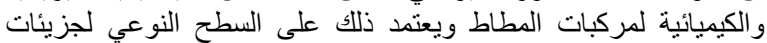
المادة المالئة و النشاط السطحي لجزيئات المطاط ولئد المادة.

$$
2.2 \text { مواد و } 2.2
$$

1 - مطاط الأكريلونترايل بوتاديئين (NBR):

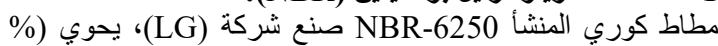

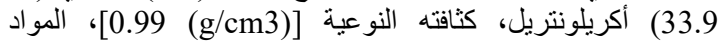

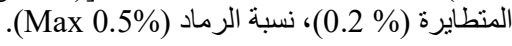

2- الكربون الأسود (Carbon Black N330):

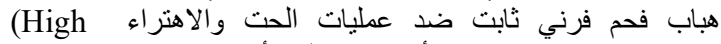
(Jsc أوكراني المنشأ صنع شركة : Abrasion Furnace) Stakhanov Carbon Black Chemical Plant) (Max 0.5\%)، النقاوة (\% (99)، مساحة سطح الادمصاص وفق الكي (CTAB absorp :82+5 m2/kg) BET 
جدول 1: النسب المو اد الداخلة في الخلطة المطّاطية

\begin{tabular}{|c|c|c|c|c|c|c|c|}
\hline Name & $\begin{array}{c}\text { C45 } \\
\text { B0 }\end{array}$ & $\begin{array}{c}\text { C45 } \\
\text { B1 }\end{array}$ & $\begin{array}{c}\text { C45 } \\
\text { B3 }\end{array}$ & $\begin{array}{c}\text { C40 } \\
\text { B5 }\end{array}$ & $\begin{array}{c}\text { C35 } \\
\text { B10 }\end{array}$ & C30 & C25 \\
B10
\end{tabular}

2. إضافة حمض الستاريك إلى العجينـة و العجن مدة دقيقة حتى

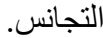
3.

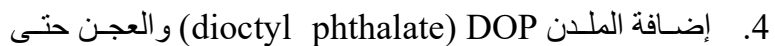

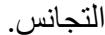
5. إضافة نصف الكمية لكل من مادة الكربون وكبريتات البـاريوم بالنسب المحددة و العجن حتى التجانس.

6. إضـافة مضـادات الأكسـدة 2,2,4-trimethyl-1,2- TMQ) dihydroquinoline) 7. إضافة نصف الأخر لكل من كمية الكربون وكبريتات الباريوم بالنسب المحددة و العجن حتى التجانس.

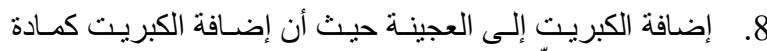

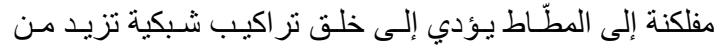

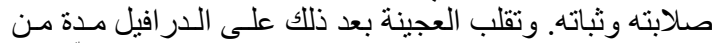

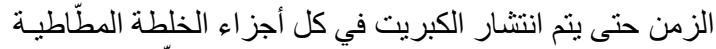

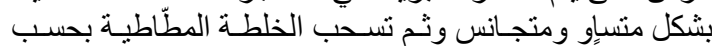

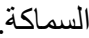

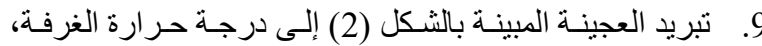

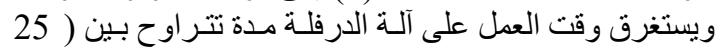

.(20-min

10. عملية الفلكنة للتر اكيب الناتجـة بوضـعها في قالب ذو تجويف

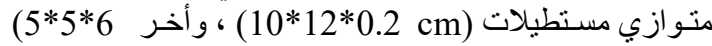
(cm)

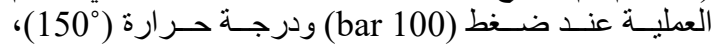
وتستغرق هذه العملية (5 min).

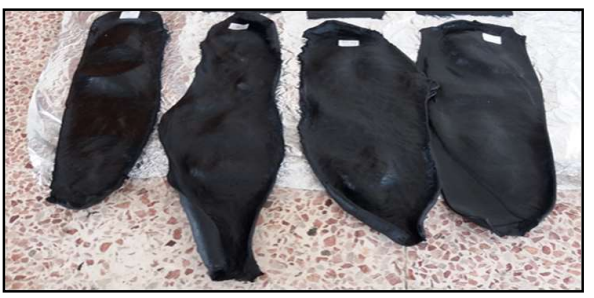

الثكل 2: الواح المطّاط المشكلة

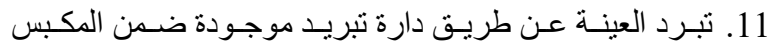

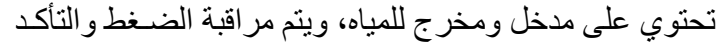

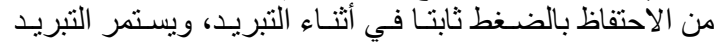

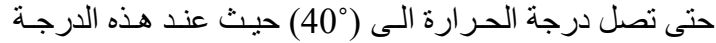
تأخذ العينة شكلها النهائي [10,1].
10- مواصفات زيت المحرك (20/50) (20)

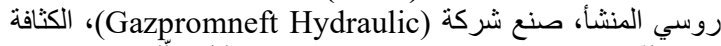

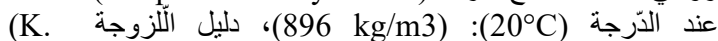
عiscosity)

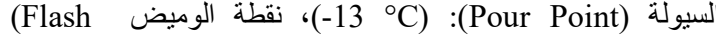

. $\left(243^{\circ} \mathrm{C}\right)$ :Point $)$

11- التولوين (Toluene) صيني المنشأ، صنع شركة (UIV CHEM)، (UIV)، سائل شفاف عديم

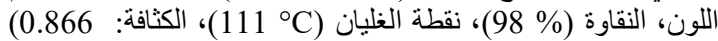

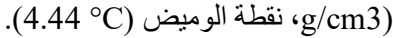

2.2.2

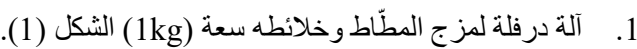

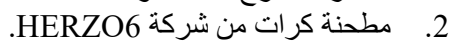
3. جهاز اختبار الثد.

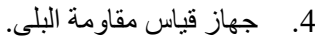
5. جهاز قياس الصلادة (Shore A). 6. 7. 8. مكبس هيدروليكي حراري من شركة (noselab ats).

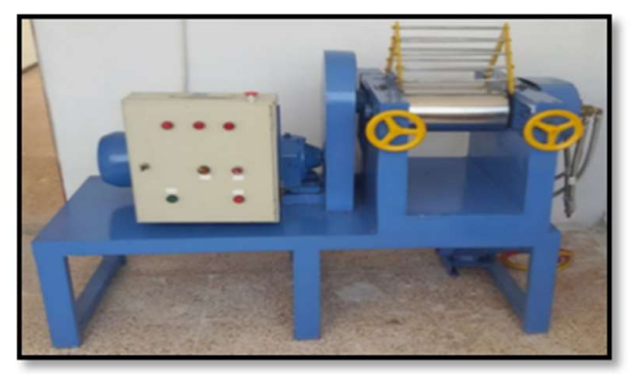

$$
\begin{aligned}
& \text { الثكل 1: آلة درفلة مزج المطّاط وخلائطه } \\
& \text { طر ائق تشكيل عينات المطّاط: }
\end{aligned}
$$

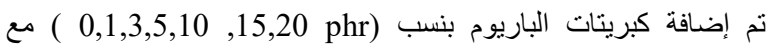

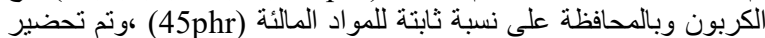

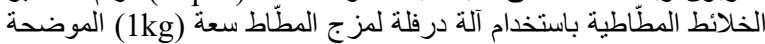

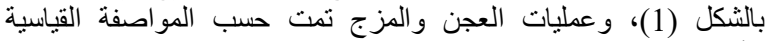

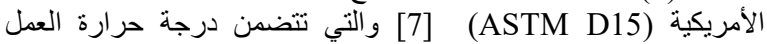
وتسلسل إضافة المواد إلى الآلة، والفترة التزمنية اللازمة للتجانس وبشة وبشكل

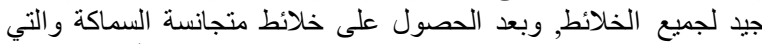

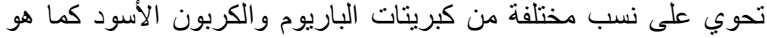

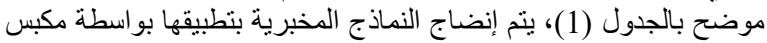

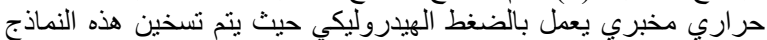

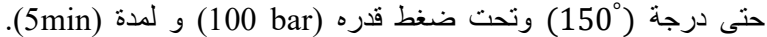

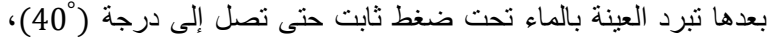
وتترك الخلائط لمدة 24 ساعة قبل اختبار ها.

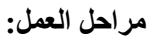

2.2.3.1

يتم تحضير الخلائط المطّاطبة وفق المر احل التالية:

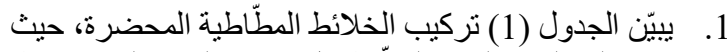

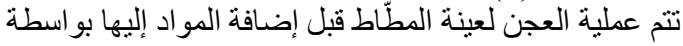

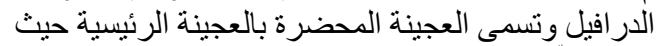

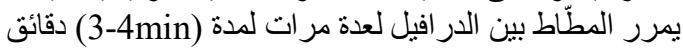

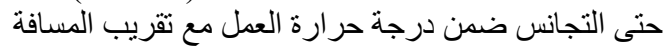

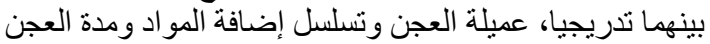
تتم وفق المو اصفة القياسية الأمريكية (ASTM D15) [7]. 


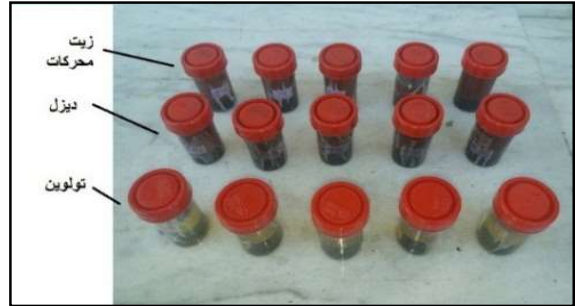

الشكل 4: العينات وقد غمرت بالمذيبات و الزيوت

2.2.4.4 اختبار مقاومة البلى (wear Resistance):

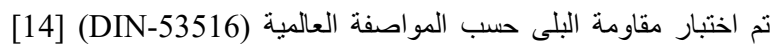

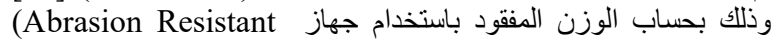
Rubber Testing Machine) كثط ورق الصنفرة و التي تؤخذ قيمتها وفق المو اصفة القياسية ISO1891)

[15art1) ثم يحسب الفاقد الحجمي المفقود (V) (15) وفق المعادلة (3):

$$
V=\frac{\Delta m * S}{\alpha * \rho}
$$

$$
\begin{aligned}
& \text { حيث: } \\
& \text { [mm³ }
\end{aligned}
$$

$$
\text { النتائسج والمناقشة: }
$$

نتائج اختبار مقاومة الثند (Tensile Test):

نتائج مقاومة الثد (Tensile strength):

2.3.1.1

يظهر الثكل (5) نتائج اختبار الثد الذي ييّين تأثير إضافة كبريتات الباريوم

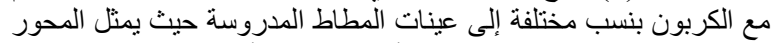

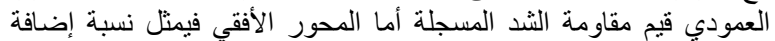

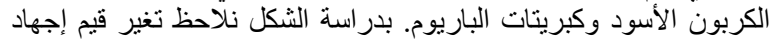

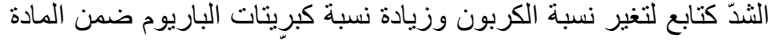

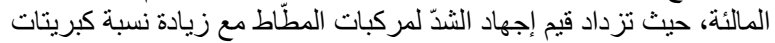

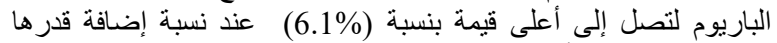

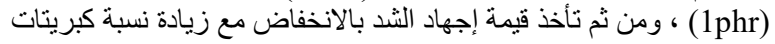

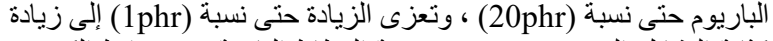

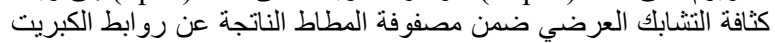

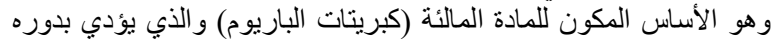

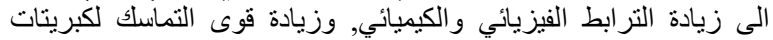

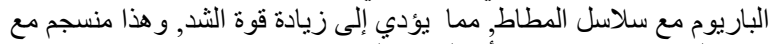
نتائج الدراسات [4] ، غير أن الزيادة المستمرة في نسبة الإضافة فوة فئل فئرة

يسبب زيادة هشاشة مركب المطاط نتيجة التحميل الز ائد من المسادة (1phr)

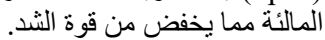

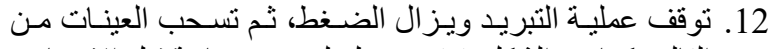
القالب كما في الثكل (3)، وتترك لمدة 24 ساعة قبل الاختبار.

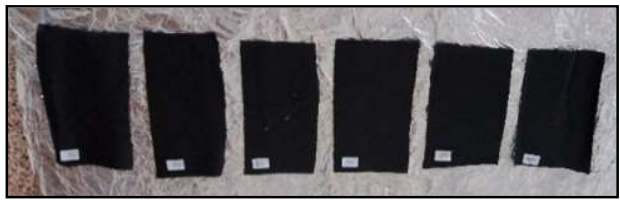

الشكل 3: الواح المطّاط بعد عملية الفلكنة

$$
\begin{array}{r}
2.2 .4 \\
\text { رختبائق أجر اء التجارب والاختبار ات: } 2.2 .4 .1
\end{array}
$$

تم هذا الاختبار باستخدام آلة الثد نوع (Testometric M350-10CT)

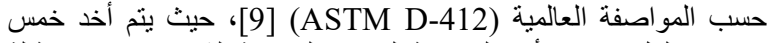
مكررات لكل اختبار و أخذ المتوسط الحسابي لمقاومة الثد (Ts) و الاستطالة

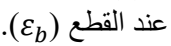

\subsubsection{2 اختبار الصلادة (Hardness):}

أجري هذا الاختبار بحسب المواصفة العالمية (DIN-53505 ) (12] )

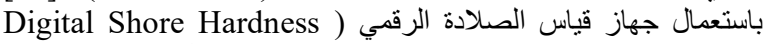

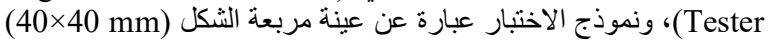
وسماكة (6 mm)، ونج).

2.2.4.3 اختبار الانتفاخ (Swelling test):

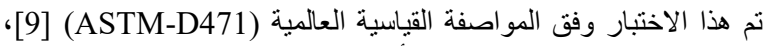

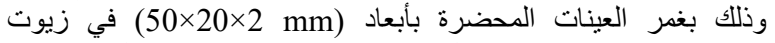

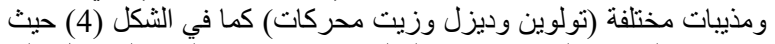
تم وزن العينات المغمورة في التولوين [3 hour 3

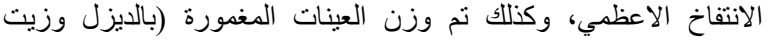

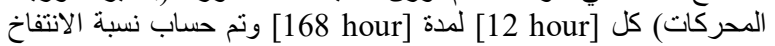

وفق المعادلة (1): (1) بل

$$
S R=\frac{W 2-W 1}{W 1} * 100 \%
$$

حيث:

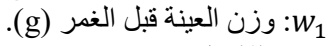
وكR : الانتفاخ : SR

وكذلك نم الحصول على كسر الجل (gel fraction) من خلأ خلال تجفيف

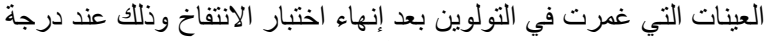

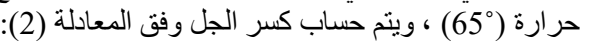

$$
g e l=\frac{W f}{W i} * 100 \%
$$

(g) وزئ (g) وزن العينة قبل الغدر: 
ويفسر ذللك لانخفاض نسبة الكربـون وبالتـالي انخفاض المسـاحة النوعيـة

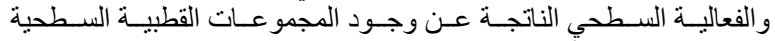

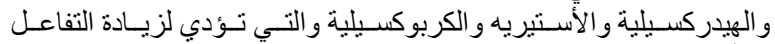

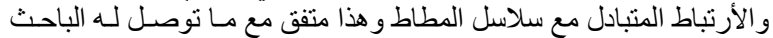

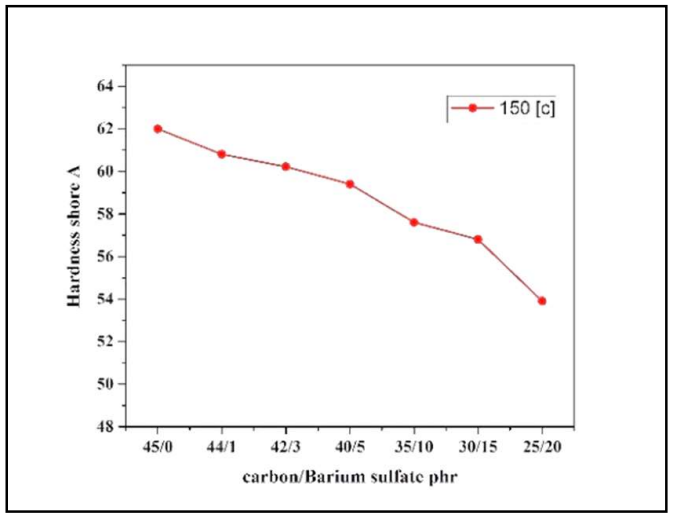

الثكل 7: تغير قيم الصلادة مع نسبة مادة الكربون الأسود-كبريتات الباريوم

2.3 .3

:(test

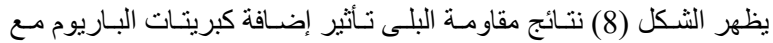

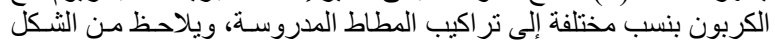

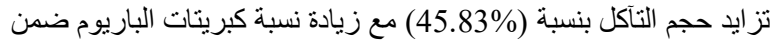

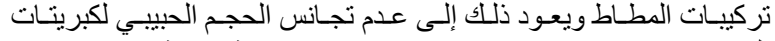

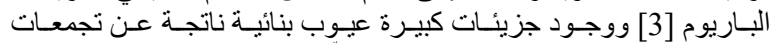

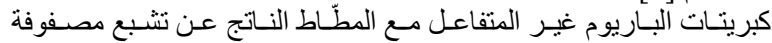

المطاط بالمادة المالئة مما يسبب زيادة فقدان الوزلئ الوزن.

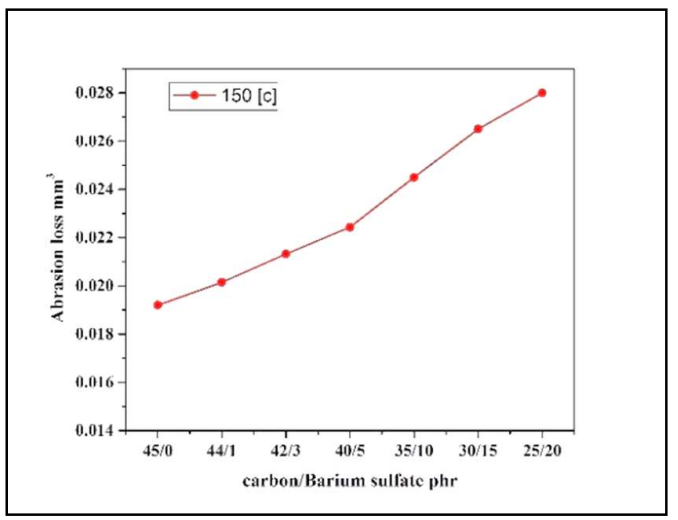

الثكل 8: تغير مقاومة البلى مع نسبة مادة الكربون الأسود-كبريتات الباريوم نينمان

2.3.4 نتائج اختبار الانتفاخ (swelling test):

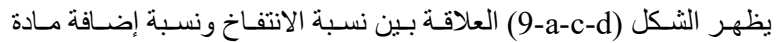

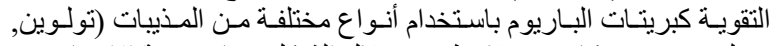

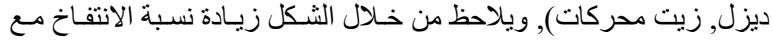

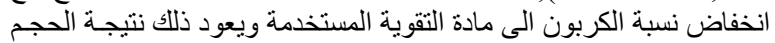

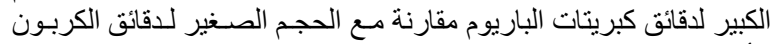

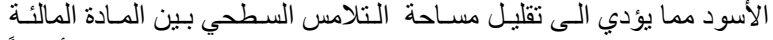

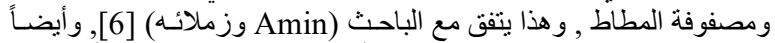
ويلاحظمن الثكل يظهر الشكل (d-9 النغ الن نسبة الانتفاخ بالتولوين أعلى من

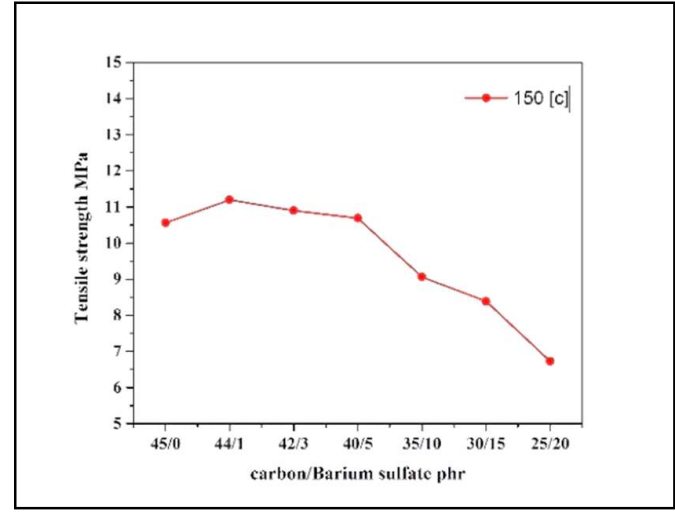

الثكل 5: تغير مقاومة الثد نسبة مادة الكربون الأسود-كبريتات الباريوم

$$
\begin{gathered}
2.3 .1 .2 \\
\text { :تائج أختبار نسبة الاستطالة عند الانقطاع (Elongation at break) }
\end{gathered}
$$

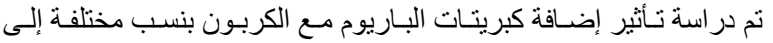

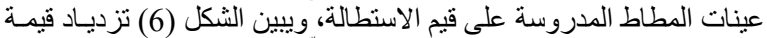

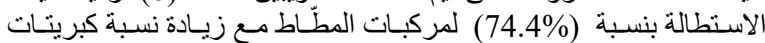

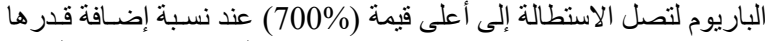

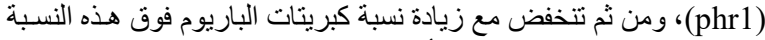

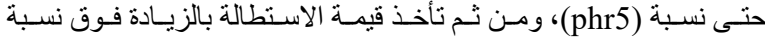

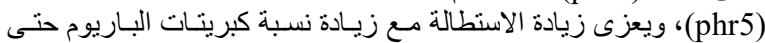

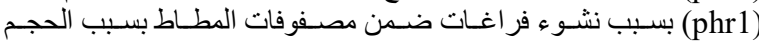

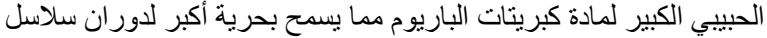

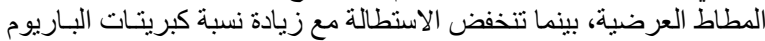

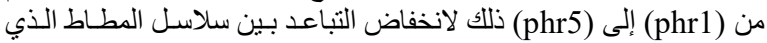

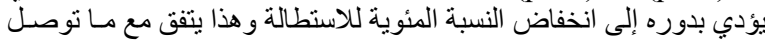

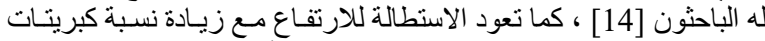

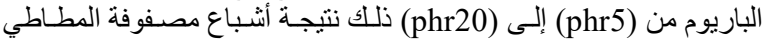

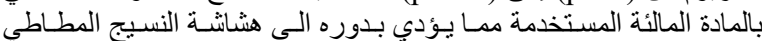
نتيجة التباعد المتز ايد بين سلاسل المطاط

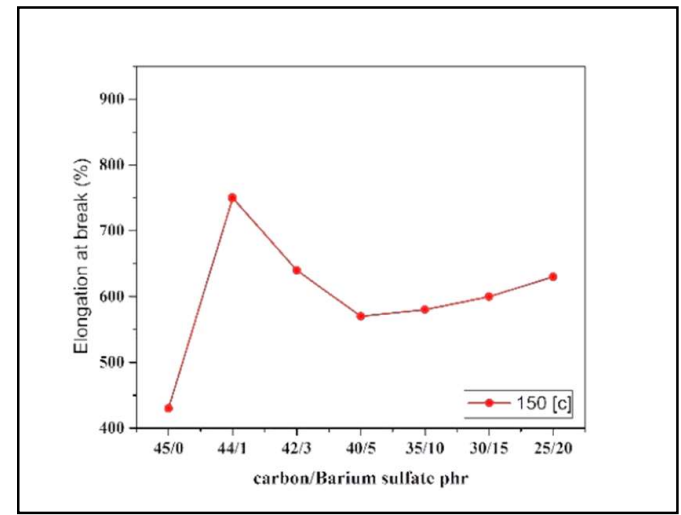

$$
\text { الثكل 6: تغير نسبة الاستطالة مع نسبة مادة الكربون الأسود-كبريتات }
$$

2.3.2 نتائج اختبار الصلادة (Hardness test):

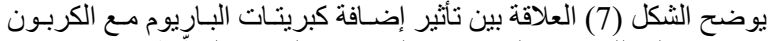

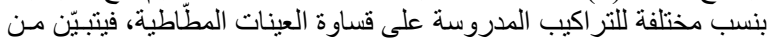

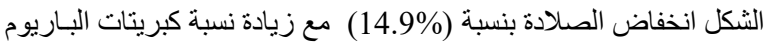

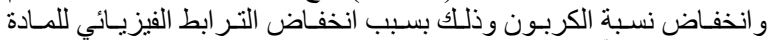

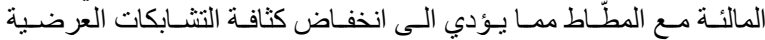
للسلاسل، فالعلاقة عكسية بين الصلادة ونسبة زيادة نسبة كبريتـات البـاريوم 


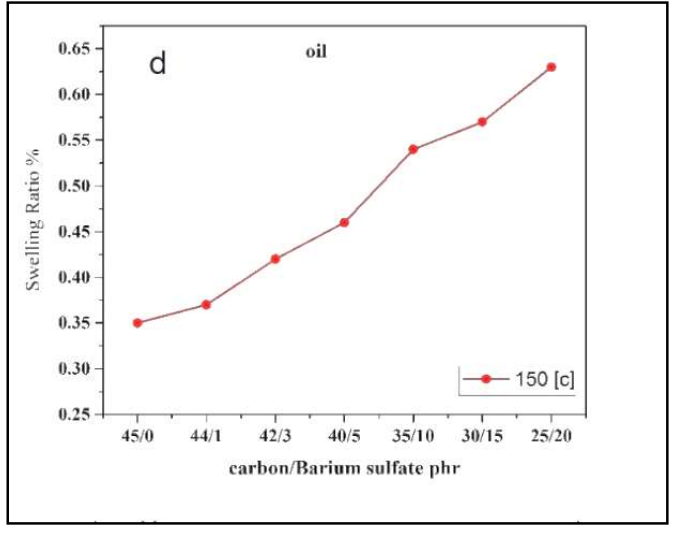

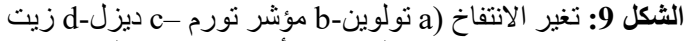

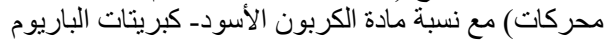

3. الاستنتاجات والتوصيات

- 3.1

1. الكربون كمادة مائلة هجينة حيث أدت ندية

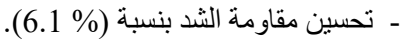

-ازدادت نسبة الاستطالة عند القطع تدريجياً بمقدار (6. (6.4).

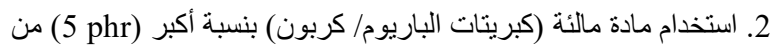

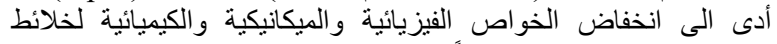
المطاط النتريلي المختلفة مقارناً بالعينة (C45B0).

3.2

1. دراسة استخدام طرائق أخرى من الفلكنة كالفلكنة بالبيروكسيد أو الفلكنة الإشعاعية.

2. دراسة استخدام حجوم مختلفة من دقائق الكربون أو مو اد تقوية أخرى.

3. دراسة استخدام كبرينات الفضة ذات الحجوم المختلفة أو النانوية.

المصادر

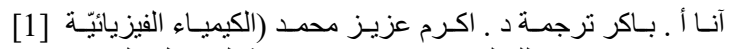
للبوليمر ات) 1984م. جامعة الموصل, العراق

كيخيا ,طارق. الكيماء الصناعية, "تكنولوجيا الصناعات الكيميائيّة [2]

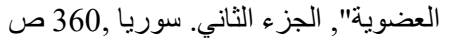

[3] Ali.A.Davoodi, Tal'at Khalkhali, Mohammad.M.Salehi, Soheil.S.Fard. Burst diaphragms based on carbon black/silica hybrid filler reinforced nitrile rubber compounds. Journal of soft matter. vol 2014, 2014, pp. 1-6.

[4] Al-maamori,M and Hamza ,A .Effect of sulfur and Nano- carbon black on the mechanical properties of hard rubber, Journal of University of Babylon, Engineering Sciences, Vol.(26), No.(2): 2018

[5] Alneamah, $M$ and Almaamori,M. Study of Thermal Stability of Nitrile Rubber/Polyimide Compounds. International Journal of Materials and Chemistry 2015, 5(1): 1-3
المذيبات الاخرى المستخدمة ويعود ذللك الى أن حجم جزيئة التولوين أصغر

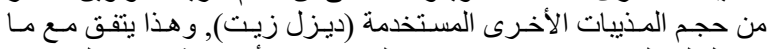

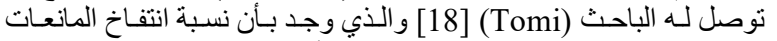

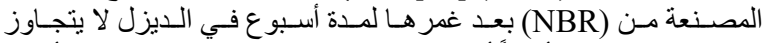

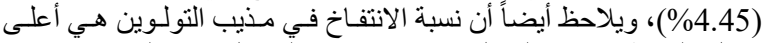

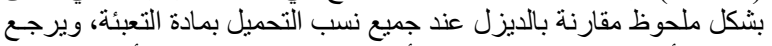
ذلك إلى أن حجم جزيئات التولوين أصغر من حجم المذيبات الأخرى.
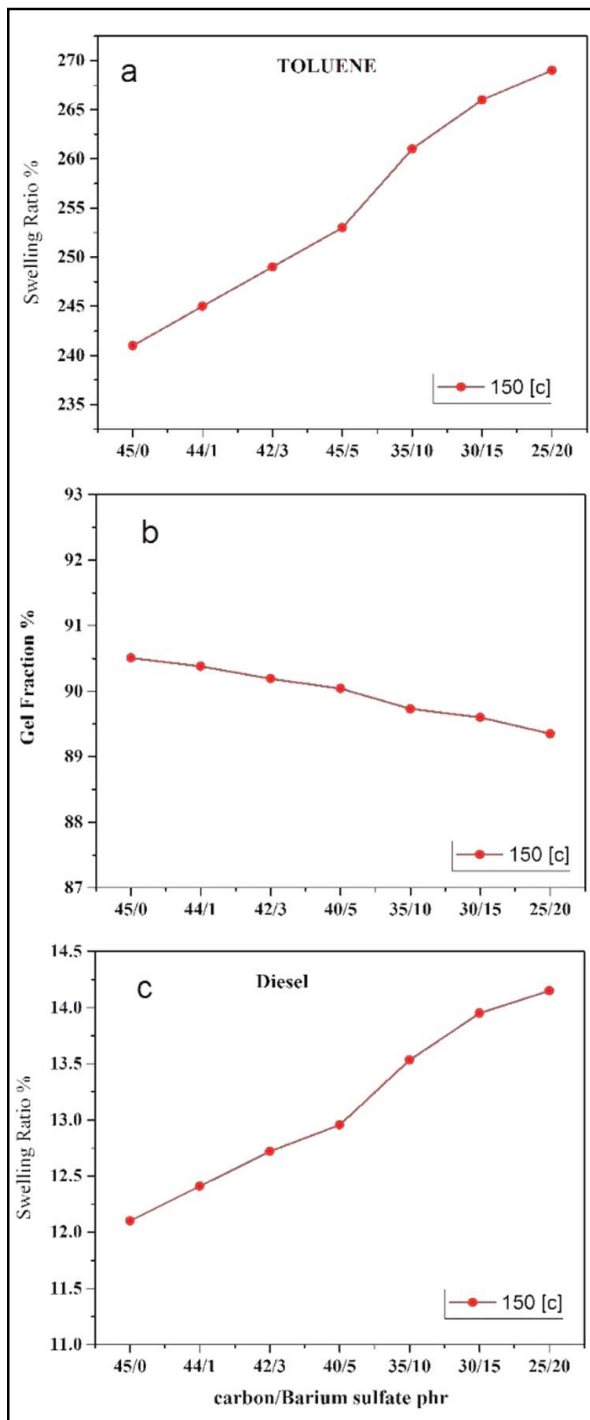

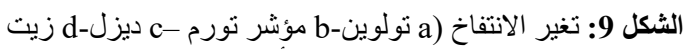

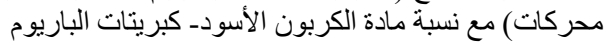


[14] Harada, M. (2016). "Analytical methods for vulcanized rubbers". International Polymer Science and Technology, vol. 43, No. 2, pp. 4554.

[15] ISO1891part1, conveyor and eleveator textile belting.

[16] Rochaa, E and Linharesa,F and , Gabrielb,C and Sousaa,A and Furtado,c . Stress relaxation of nitrile rubber composites filled with a hybrid metakaolin/carbon black filler under tensile and compressive, Applied Clay Science 151 (2018) $181-188$

[17] Senthilvel ,K and Vishvanathperumal ,S and Prabu ,B and John Baruch .Studies on the Morphology, Cure Characteristics and Mechanical. Polymers \& Polymer Composites, Vol. 24, No. 7, 2016.

[18] Tomi Juselius., Effect of Diesel Fuel Composition on Sealing Materials., Metropolia University of Applied Sciences Bachelor of Engineering Automotive Engineering Thesis, March 3, 2018

[19] YASIN, Tariq, et al. Radiation vulcanization of acrylonitrile-butadiene rubber with polyfunctional monomers. Reactive and Functional Polymers, 2002, 53.2-3: 173-181.
[6] Amin, M and Nadras, O. Comparative Study of Bentonite Filled Acrylonitrile Butadiene Rubber and Carbon Black Filled NBR Composites Properties International Journal of Automotive and Mechanical Engineering. Volume 15, Issue 3 pp. 5468-5479 Sept 2018.

[7] ASTM D15 - 72, methods of compound and sample preparation for physical testing of rubber products.

[8] ASTM D412 - 16, Standard Test Methods for Vulcanized Rubber and Thermoplastic Elastomers-Tension.

[9] ASTM D471, Standard Test Method for Rubber Property-Effect of Liquids.

[10] Chache M, Ali A, Makhlouf A."Design a model rolling machine to mix rubber and its components". Tartous University, 2016.

[11] Chache M, Ali A, Makhlouf A."Study physicomechanical and chemical properties of mixtures of natural rubber \& thermal cement" Tishreen University Journal-Engineering Sciences Series, 38.2 (2017).

[12] DIN 53505, Shore A and Shore D hardness testing of rubber.

[13] DIN 53516, Testing of rubber and elastomers, determination of abrasion resistance.

\title{
The effect of barium sulfate as a filler on the physical and mechanical properties of nitrile rubber blends
}

\author{
Ali Alhaidar ${ }^{1, *}$, Ali Ali $^{2}$ and Mayssa Shash ${ }^{3}$ \\ 'Department of Machines and Equipments, Faculty of Technical Engineering, Tartous University, Syria, ali.alhaidar315@gmail.com \\ ${ }^{2}$ Department of Food Technology, Faculty of Technical Engineering, Tartous University, Syria, alimali1968@yahoo.com \\ ${ }_{3}^{3}$ Department of Machines and Equipments, Faculty of Technical Engineering, Tartous University, Syria, mayssaali@yahoo.fr \\ *Correspondeng auther :Ali Alhaidar,email: alialhaidar315@gmail.com \\ Published online: 30 June 2021
}

Abstract :In this study, the effect of adding barium sulfate as a filler in the properties of NBR has been studied by preparing blends of rubber containing different proportions of barium sulfate $(0,1,3,5,10,15,20 \mathrm{phr})$, on two roll mill then describe Physio - mechanical and chemical properties of the prepared blends were described by testing them for tensile strength and abrasion resistance, value of hardness and swelling in different solvents. The results showed an increase in tensile strength by $(6.1 \%)$ with an increase in the percentage of barium sulfate within the rubber mixtures until (1phr), then the tensile strength decreased with an increase in the ratio of barium sulfate in the rubber compositions over (1phr). As for elongation at break, it gradually increased by $(74.4 \%)$ at the rate of adding ( $1 \mathrm{phr})$ and then decreased with increasing the percentage of barium sulfate to (phr5) and then increased with the increase of barium sulfate. While the hardness values decreased by $(14.9 \%)$ with the increase in the percentage of barium sulfate to the ratio of (phr20), friction resistance also decreased by $(45.83 \%)$ with an increase in the percentage of barium sulfate to (phr20). The results of the study also showed that the productivity of samples containing black carbon only (C45B0) was very low compared to samples containing barium sulfate.

Keywords: Vulcanization, barium sulfate, Rubber, Carbon black, rolling 